\title{
Utilization of FDG-PET/CT in the diagnosis of native valve endocarditis: There is a hope, but we need more data!
}

\author{
Ayaz Aghayev, MD \\ a Cardiovascular Imaging, Department of Radiology, Brigham and Women's Hospital, Harvard \\ Medical School, Boston, MA
}

Received Apr 13, 2020; accepted Apr 13, 2020

doi: $10.1007 / \mathrm{s} 12350-020-02308-9$

\section{See related article, https://doi.org/10.10 07/s1 2350-020-02092-6.}

Infective endocarditis (IE) diagnosis and management require a multidisciplinary approach, and cardiac imaging plays an important role. Echocardiographyboth transthoracic echocardiography (TTE) and transesophageal echocardiography (TEE) — is a cornerstone in the diagnosis of patients with IE as a part of the Modified Duke Criteria. ${ }^{1}$ However, echocardiography findings could be equivocal or difficult to interpret in some instances. In 2015, the American Heart Association (AHA) and European Society of Cardiology (ESC) published documents-the AHA acknowledged the potentials of advanced imaging modalities, such as fluorodeoxyglucose positron emission tomography/computed tomography (FDG-PET/CT), and cardiac CT, with no recommendation; however, the ESC recommended the utilization of both FDG-PET/CT and cardiac CT in the diagnosis of IE. ${ }^{2,3}$ As per the 2015 ESC guidelines, both FDG-PET/CT and cardiac CT have the potentials in reclassifying the "possible IE", category to the "definite IE" category and in the detection of septic embolic foci throughout the body. ${ }^{3}$ In particular, the 2015 ESC guidelines recommended the use of FDG-PET/CT along with cardiac CT in patients with possible prosthetic heart valve endocarditis (PVE) as per the Modified Duke Criteria. Since these

Reprint requests: Ayaz Aghayev, MD, Cardiovascular Imaging, Department of Radiology, Brigham and Women's Hospital, Harvard Medical School, 75 Francis St, Boston, MA 02115; Aaghayev@bwh.harvard.edu

J Nucl Cardiol 2022;29:3455-7.

$1071-3581 / \$ 34.00$

Copyright (C) 2020 American Society of Nuclear Cardiology. recommendations, a growing body of literature investigated the use of FDG-PET/CT in the diagnosis of IE.

Most of the literature is on the use of FDG-PET/CT for PVE. Saby et al were one of the first groups to analyze FDG-PET/CT in patients with PVE. ${ }^{4}$ In this prospective study of 72 patients with suspected PVE, FDG-PET/CT increased the sensitivity of the Modified Duke Criteria from $70 \%$ to $97 \%$, which was a result of the significant reduction in the number of patients with " possible PVE.' Since then, multiple small studies were conducted with similar results, and a meta-analysis by Mahmood et al in 2017 demonstrated pooled sensitivity of $80.5 \%$ and specificity of $73.1 \% .^{5}$ In 2018, a large, retrospective, multicenter cohort by Swart et al showed that sensitivity and specificity of PVE diagnosis were $74 \%$ and $91 \%$, respectively. Interestingly, when the authors excluded confounders-patients with low inflammatory activity (C-reactive protein $<40 \mathrm{mg} / \mathrm{L}$ ) at the time of imaging and the use of surgical adhesives during prosthetic heart valve implantation-then the sensitivity and specificity to diagnose PVE were as high as $91 \%$ and $95 \%$, respectively. And most recently, in 2019, de Camargo et al reported sensitivity and specificity of $93 \%$ and $90 \%$, respectively, in a large singlecenter cohort of 188 patients. ${ }^{6}$ Multimodality imaging with FDG-PET/CT and cardiac CT is proven to be even more valuable, which can increase the sensitivity and specificity from $86.4 \%$ and $87.5 \%$, respectively, for FDG-PET/CT only to $91 \%$ and $90.6 \%$, respectively, for FDG-PET/CT and cardiac CT together. ${ }^{7}$

In contrast to PVE diagnosis with FDG-PET/CT, there is scarce literature on the use of FDG-PET/CT in the diagnosis of native valve endocarditis (NVE). The observed sensitivity in diagnosing of NVE with FDGPET/CT was significantly lower compared with that in diagnosing of PVE. ${ }^{6,8}$ A recent study by de Camargo et al, in 2019, with a large cohort of 115 patients with suspected NVE reported sensitivity and specificity of 
$22 \%$ and $100 \%$, respectively. ${ }^{6}$ The EURO-ENDO registry, a prospective observational cohort of 3116 patients across 40 countries and 156 centers demonstrated that less than $10 \%$ of the patients with suspected NVE underwent FDG-PET/CT. ${ }^{9}$ And the sensitivity in this study of the confirmed NVE was $28 \%$ compared with $67 \%$ for PVE. A possible explanation for the low sensitivity in these studies could be due to (1) small-size $(<$ $10 \mathrm{~mm}$ ) vegetation on the valves, which could be challenging to detect due to low spatial and temporal resolutions of the modality; (2) similarly small-size vegetations, meaning they are less vascular or avascular lesions (therefore, there is minimal or no FDG tracer deposition within these vegetations); (3) timing of the FDG-PET/CT (it has been shown that during the subacute or chronic phase, vegetations in the native valve demonstrate less inflammatory cells and higher fibrosis compared with those in prosthetic valves). ${ }^{6}$ High specificity in NVE diagnosis with FDG-PET/CT could indicate that those valve infections are severely complicated, such as perivalvular abscess formation. Both large studies by de Camargo et al and Kouijzer et al suggested that negative FDG-PET/CT results should not be used to exclude NVE; however, given the high specificity, in selected cases-sustained suspicion of NVE-FDG-PET/CT can be used to assess uptake around the valve. Besides, PET/CT is also an essential tool for the detection of metastatic infection. ${ }^{6,8}$ As readers would agree with the aforementioned limitations of FDG-PET/CT on NVE cases, given the lack of data, it is difficult to conclude that it is not a useful modality. Therefore, studies like in this issue of the Journal of Nuclear Cardiology by Abikhzer et al are important additions to the literature (Abikhzer).

In this study, the authors demonstrated a retrospective study assessing the use of FDG-PET/CT for the diagnosis of NVE. They evaluated 54 suspected NVE patients using FDG-PET/CT, where 31 patients had confirmed NVE. FDG-PET/CT results were positive in 21 out of the 31 patients with sensitivity and specificity of $68 \%$ and $100 \%$, respectively. Although the number of patients is small, this is so far the highest published sensitivity in the diagnosis of NVE in the medical literature. One of the main reasons to have false-negative results is the initiation of antibiotic therapy before the imaging study, and the other one is incomplete myocardial suppression with a high-fat-low-carbohydrate diet. As expected, when they excluded patients with incomplete myocardial suppression, the sensitivity went up to $80 \%$ without any reduction in specificity. The confounders, including longer antibiotic use, incomplete myocardial suppression with prior diet, the timing of surgery, and the utilized surgical materials, have been well described in PVE, which can lead to misdiagnosis. ${ }^{10,11}$ However, the readers would agree that two of these confounders are essential in NVE interpretation with FDG-PET/CT-prolonged use of antibiotics before the procedure and incomplete suppression of the myocardium with proper diet-and should be taken into consideration.

As mentioned above, one of the main reasons for the low sensitivity of FDG-PET/CT in NVE diagnosis is the vegetation size. Intuitively, smaller size vegetations will have less inflammation, less inflammatory cells, and thus less FDG uptake. Along with these, in this study, the authors found a significant difference in vegetation sizes in patients with false-negative vs true-positive results $(9.6 \pm 5.9 \mathrm{~mm}$ vs $14.4 \pm 6.1, P=0.049)$.

Another interesting point, which readers will find, is the reclassification of patients using the Modified Duke Criteria after utilization of FDG-PET/CT. A total of eight "possible IE" patients were reclassified from "possible IE" to "definite IE," and all of these patients had the final diagnosis of NVE. The Modified Duke Criteria sensitivity to NVE diagnosis increased from $48 \%$ to $77 \%$ after the utilization of FDG-PET/CT. This pattern is similar to PVE endocarditis; however, there is a need for more data on this.

Lastly, the authors would like to draw attention to one technical aspect of this study. Although it was not part of their routine practice, they have acquired delayed imaging for some patients. As expected, the maximum standardized uptake values (SUVmax) remained unchanged; however, target-to-background ratios were higher on delayed images attributed to the decrease in blood pool activity, and mildly FDG-avid lesions were better seen on delayed images. High blood pool activity can mask small, mildly FDG-avid vegetations and thus will result in false-negative results. Delayed image acquisition could be promising, and these images can be obtained in NVE cases. Particularly extra electrocardiogram (ECG)-gated cardiac bed acquisition as delayed imaging, after whole-body FDG-PET/CT, can give more information in NVE cases. However, it should be interpreted carefully, since it has been shown that delayed imaging could result in false-positive results in patients with PVE. ${ }^{12}$

One of the major caveats in this and some of the previous IE studies is the lack of multimodality imaging-correlation with other imaging modalities, particularly ECG-gated cardiac CT, which could improve diagnosis performance. Also, FDG-PET/CT, along with cardiac $\mathrm{CT}$, could be an alternative imaging modality, where TEE cannot be performed (i.e., esophageal atresia) or especially during this time where coronavirus disease-2019 (COVID-19) pandemic is severely affecting the condition of healthcare services. FDG-PET/CT and cardiac CT could be an alternative for 
COVID-19 patients in order to reduce the exposure risk to echocardiography personnel.

Although this study demonstrates the usefulness of FDG-PET/CT in NVE, with the highest sensitivity in the literature, the medical community still need more data and large studies. In addition, future studies should focus on developing a guideline regarding the standardized protocols for patient preparation and image acquisitions, semi-quantitative measurements, and image interpretation, which can improve the clinical care of patients with NVE. ${ }^{13}$

\section{References}

1. Li JS, Sexton DJ, Mick N, Nettles R, Fowler Jr VG, Ryan T, et al. Proposed modifications to the Duke criteria for the diagnosis of infective endocarditis. Clin Infect Dis 2000;30:633-8.

2. Baddour LM, Wilson WR, Bayer AS, Fowler Jr VG, Tleyjeh IM, Rybak MJ, et al. Infective endocarditis in adults: Diagnosis, antimicrobial therapy, and management of complications: A scientific statement for healthcare professionals from the American Heart Association. Circulation 2015;132:1435-86.

3. Habib G, Lancellotti P, Antunes MJ, Bongiorni MG, Casalta JP, Del Zotti F, et al. 2015 ESC Guidelines for the management of infective endocarditis: The Task Force for the Management of Infective Endocarditis of the European Society of Cardiology (ESC). Endorsed by: European Association for Cardio-Thoracic Surgery (EACTS), the European Association of Nuclear Medicine (EANM). Eur Heart J 2015;36:3075-128.

4. Saby L, Laas O, Habib G, Cammilleri S, Mancini J, Tessonnier L, et al. Positron emission tomography/computed tomography for diagnosis of prosthetic valve endocarditis: Increased valvular $18 \mathrm{~F}$ fluorodeoxyglucose uptake as a novel major criterion. J Am Coll Cardiol 2013;61:2374-82.

5. Mahmood M, Kendi AT, Ajmal S, Farid S, O'Horo JC, Chareonthaitawee $\mathrm{P}$, et al. Meta-analysis of 18F-FDG PET/CT in the diagnosis of infective endocarditis. J Nucl Cardiol 2017;26:92235 .
6. de Camargo RA, Bitencourt MS, Meneghetti JC, Soares J, Gonçalves LFT, Buchpiguel CA, et al. The role of 18F-FDG-PET/CT in the Diagnosis of left-sided Endocarditis: Native vs. prosthetic valves endocarditis. Clin Infect Dis 2019.

7. Pizzi MN, Roque A, Fernandez-Hidalgo N, Cuéllar-Calabria H, Ferreira-González I, Gonzàlez-Alujas MT, et al. Improving the diagnosis of infective endocarditis in prosthetic valves and intracardiac devices with $18 \mathrm{~F}$-fluordeoxyglucose positron emission tomography/computed tomography angiography: Initial results at an Infective Endocarditis Referral Center. Circulation 2015;132:1113-26.

8. Kouijzer IJE, Berrevoets MAH, Aarntzen E, De Vries J, Van Dijk AP, Oyen WJ, et al. 18F-fluorodeoxyglucose positron-emission tomography combined with computed tomography as a diagnostic tool in native valve endocarditis. Nucl Med Commun 2018;39:747-52.

9. Habib G, Erba PA, Iung B, Donal E, Cosyns B, Laroche C, et al. Clinical presentation, aetiology and outcome of infective endocarditis. Results of the ESC-EORP EURO-ENDO (European infective endocarditis) registry: A prospective cohort study. Eur Heart J 2019;40:3222-32.

10. Swart LE, Gomes A, Scholtens AM, Sinha B, Tanis W, Lam MG, et al. Improving the diagnostic performance of (18)F-fluorodeoxyglucose positron-emission tomography/computed tomography in prosthetic heart valve endocarditis. Circulation 2018;138:1412-27.

11. Scholtens AM, Swart LE, Verberne HJ, Tanis W, Lam MG, Budde RP. Confounders in FDG-PET/CT imaging of suspected prosthetic valve endocarditis. JACC Cardiovasc Imaging 2016;9:1462-5.

12. Scholtens AM, Swart LE, Verberne HJ, Budde RPJ, Lam M. Dualtime-point FDG PET/CT imaging in prosthetic heart valve endocarditis. J Nucl Cardiol 2018;25:1960-7.

13. Scholtens AM, Swart LE, Kolste HJT, Budde RPJ, Lam M, Verberne HJ. Standardized uptake values in FDG PET/CT for prosthetic heart valve endocarditis: A call for standardization. J Nucl Cardiol 2018;25:2084-91.

Publisher's Note Springer Nature remains neutral with regard to jurisdictional claims in published maps and institutional affiliations. 